\title{
Desenvolvimento bucal e aleitamento materno em crianças com fissura labiopalatal
}

\author{
Oral development and breastfeeding in children with cleft lip and palate
}

\author{
Luciana Rodrigues V. Batista ${ }^{1}$, Thaisa Cezária Triches², Emilia Addison M. Moreira ${ }^{3}$
}

RESUMO

Objetivo: Aspectos funcionais do estado de saúde bucal de crianças portadoras de fissuras de lábio e/ou palato interferem na capacidade natural de serem adequadamente alimentadas, como também em seu crescimento e desenvolvimento. Essa revisão visou verificar a influência do aleitamento materno sobre o desenvolvimento buco-maxilo-facial em crianças com fissuras labiais.

Fontes de dados: Revisão das bases de dados Medline e SciELO entre 2000 e 2010, utilizando os termos: "fenda labial", "fissura palatina”, "aleitamento materno", "alimentação artificial” e "higiene bucal". As informações foram agrupadas, de modo a estabelecer a relação entre a amamentação natural e artificial no desenvolvimento bucofacial e estado de saúde bucal.

Síntese dos dados: A pesquisa inicial selecionou um total de 27.046 artigos, dos quais 26.793 abordaram o aleitamento materno e a alimentação artificial, 119 relacionavam a influência do aleitamento materno sobre o desenvolvimento bucofacial, enquanto 134 analisaram a relação entre a amamentação e o estado de saúde bucal. Foram selecionados para a análise 34 artigos, sendo 20 com crianças portadoras de fendas labiais e/ou fissuras palatinas.

Conclusões: $\mathrm{O}$ aleitamento natural em crianças portadoras de fissuras de lábio e/ou palato é fator decisivo para a correta maturação e crescimento craniofacial em nível ósseo, muscular e funcional e na prevenção de problemas bucais. Além disso, essas crianças apresentam maior prevalência de cárie dental, problemas relacionados à doença periodontal, podendo ainda apresentar problemas de má oclusão.

Palavras-chave: fenda labial; fissura palatina; aleitamento materno; alimentação artificial; higiene bucal.

Instituição: Universidade Federal de Santa Catarina (UFSC), Florianópolis, SC, Brasil

'Doutoranda em Odontologia pela UFSC, Florianópolis, SC, Brasil 2Doutoranda em Odontologia pela UFSC, Florianópolis, SC, Brasil; Bolsista do Programa de Apoio ao Plano de Reestruturação e Expansão das Universidades Federais da Coordenação de Aperfeiçoamento de Pessoal de Nível Superior (Capes)

${ }^{3}$ Doutora em Ciências dos Alimentos pela Universidade de São Paulo (USP); Professora do Programa de Pós-Graduação em Nutrição e Odontologia da UFSC, Florianópolis, SC, Brasil

\section{ABSTRACT}

Objective: Functional aspects of oral health of children with cleft lip and/or palate interfere in their possibility of being naturally fed, as well as in their growth and development. This review aimed to verify the influence of breastfeeding on orofacial development of children with cleft lip and/or palate.

Data sources: Data were obtained by literature search (2000-2010) of Medline and SciELO databases, using as key-words "cleft lip", "cleft palate", "breast feeding", "complementary feeding" and "oral hygiene". Information was grouped in order to establish the relationship between natural breastfeeding and complementary feeding with orofacial development and oral health status.

Data synthesis: Initial search retrieved 27,046 articles and in 26,793 of them, the subject was breastfeeding and complementary feeding. Among those, there were 119 about the influence of breastfeeding on orofacial development and 134 about the relationship between breastfeeding and oral health status; 34 articles were selected and in 20 children with cleft lip and/or palate were enrolled.

Conclusions: Breastfeeding in children with cleft lip and/or palate is a decisive factor not only for correct craniofacial maturation and growth at bone, muscular and functional levels, but also for the prevention of oral problems. These children present higher prevalence of dental caries, periodontal diseases, as well as occlusion related problems.

Key-words: cleft lip; cleft palate; breast feeding; bottle feeding; oral hygiene.
Endereço para correspondência:

Emília Addison M. Moreira

Departamento de Nutrição do Centro de Ciências da Saúde da UFSC, Campus Universitário, s/n - Trindade

CEP 88040-970 - Florianópolis/SC

E-mail: addison@ccs.ufsc.br

Conflito de interesse: nada a declarar

Recebido em: 9/6/2010

Aprovado em: 4/4/2011 


\section{Introdução}

As fissuras labiopalatais constituem malformações congênitas com incidência, no Brasil, de uma em cada 650 nascimentos ${ }^{(1)}$. A descontinuidade das estruturas do lábio, do palato ou de ambos ${ }^{(1,2)}$ pode alterar o estado nutricional do recém-nascido devido à interferência em sua capacidade natural de se alimentar ${ }^{(3,4)}$.

As fissuras podem ser de vários tipos: fissura pré-forame incisivo completa ou incompleta (unilateral, bilateral, mediana); fissura transforame incisivo (unilateral, bilateral, mediana); fissura pós-forame incisivo (completa ou incompleta) e fissuras raras da face ${ }^{(5)}$. Com relação ao estado de saúde bucal dos portadores de fissuras, independente do tipo, os autores são unânimes em relatar a presença de comprometimento, com maior risco de cárie dentária ${ }^{(6-10)}$. Esse fato decorre não somente da anatomia da região, como também de fatores relacionados à colonização pelo micro-organismo mutans, ao tipo e consumo de carboidrato, a horário e frequência da alimentação e à higiene bucal ${ }^{(11-13)}$.

A fissura não exclui o aleitamento materno ${ }^{(14)}$ nem a alimentação, uma vez que os reflexos de sucção e deglutição se encontram preservados ${ }^{(1)}$. A amamentação natural tem grande importância para o desenvolvimento facial. A literatura atual quase não aborda a questão da amamentação e/ou alimentação e estado de higiene bucal das crianças portadoras de fissuras labiopalatais. Dessa forma, este estudo teve como objetivo investigar, por meio de revisão da literatura, a relação entre o desenvolvimento bucofacial e o aleitamento natural ou artificial em crianças com fissura labiopalatal.

\section{Método}

Os artigos selecionados na presente revisão foram pesquisados nas bases de dados Medline e ScIELO, considerando o período de 2000 a 2010. Foram utilizados os termos: "aleitamento materno", "alimentação", "fenda labial”, "fissura palatina", "higiene bucal”; e "breast feeding", "feeding", "cleft lip", "cleft palate", "oral hygiene". Na análise das publicações, as informações foram agrupadas de modo a estabelecer a relação entre a amamentação natural e artificial no desenvolvimento bucofacial e estado de saúde bucal. A pesquisa inicial selecionou um total de 27.046 artigos, dos quais 26.793 versavam sobre o aleitamento materno e alimentação artificial, 119 relacionavam a influência do aleitamento materno no desenvolvimento bucofacial, enquanto 134 revelavam a relação entre a amamentação e o estado de saúde. Foram selecionados para a análise 34 artigos, sendo que 20 envolviam crianças com fendas labiais e/ou fissuras palatais.

\section{Síntese dos dados}

Os dados levantados na revisão da literatura foram agrupados em subitens com o objetivo de sistematizar os achados de acordo com a influência da amamentação natural no desenvolvimento bucofacial, o adequado manuseio no processo de aleitamento artificial e a relação entre amamentação e estado de saúde bucal. Na Tabela 1 foram relacionados os estudos mais relevantes.

\section{A amamentação natural no desenvolvimento bucofacial}

Em decorrência do rápido crescimento durante o primeiro ano de vida, a infância torna-se um dos períodos mais críticos do ciclo vital. Dessa forma, a nutrição e, mais especificamente, a alimentação natural, são essenciais para o desenvolvimento humano ${ }^{(4)}$.

O desenvolvimento da dentição e suas estruturas de suporte podem se iniciar logo após o nascimento, por meio da amamentação. O processo de deglutição na amamentação, pelo exercício da musculatura e o correto crescimento facial, permite o desenvolvimento normal da primeira dentição. O mecanismo pelo qual a amamentação contribui para a formação da dentição ocorre, primeiro, pela apreensão do mamilo, vedando a cavidade bucal, e, segundo, pela pressão do mamilo para a sucção do leite. Há a formação de vácuo consequente à vedação realizada e à contração do mamilo pela língua, favorecendo a extração do leite do seio para que o mesmo seja deglutido, levando a mandíbula à sua posição original ${ }^{(15,16)}$.

$\mathrm{O}$ aleitamento materno tem muitas vantagens ${ }^{(2,4,16,17)}$, principalmente para os portadores de fissura, pois, o ato de sugar com mais força favorece o desenvolvimento da musculatura da face e aumenta a força dos movimentos executados com a língua ${ }^{(18)}$. A amamentação desenvolve o sistema estomatognático, pelo equilíbrio das forças musculares, estimulando o crescimento anteroposterior da mandíbula e reforçando o circuito neural fisiológico da respiração ${ }^{(19)}$. $\mathrm{Na}$ amamentação natural, a criança aprende a posicionar corretamente a língua, ganhando tônus e oclusão adequados, afastando hábitos, como sucção de chupeta e digital. O tempo de sucção no peito é muito maior do que na mamadeira e o uso da mamadeira pode levar à falta de satisfação, impulso que poderá levar o lactente a sugar o dedo, o lábio ou a própria língua. Assim, quando se faz uso exclusivo da mamadeira, 
deixa de haver estímulo para o crescimento anteroposterior da mandíbula, pois a criança não precisa realizar o exercício muscular de protrusão e retrusão mandibular ${ }^{(16)}$.

O tipo de aleitamento da criança, a orientação prévia sobre amamentação natural às mães, assim como a instalação de hábitos de sucção não nutritiva e a presença de má oclusão foram avaliados em 79 crianças, de ambos os sexos, com idades entre dois e cinco anos. Observou-se existir uma relação entre o prolongamento do aleitamento materno e a redução da instalação de hábitos de sucção; a orientação prévia das mães sobre amamentação resultou em prolongamento no tempo de aleitamento natural; crianças com hábitos bucais tiveram maior risco relativo de desenvolver má oclusão ${ }^{(20)}$.

Os lactentes com fissura palatal apresentam alguns problemas relacionados à amamentação, uma vez que o leite costuma passar pelo defeito palatal e penetrar na cavidade nasal, podendo ser aspirado nos pulmões. Além disso, o defeito dificulta a sucção eficiente do lactente, favorecendo o vômito, o que pode agravar o estado nutricional da criança fissurada e levar a outros problemas, como o engasgo durante as mamadas e até a asfixia ${ }^{(21)}$. Apesar disso, existem crianças fissuradas que conseguem sugar o peito materno ${ }^{(1,7,22)}$.

Tabela 1 - Amamentação, desenvolvimento bucofacial e estado de saúde bucal

\begin{tabular}{|c|c|c|c|}
\hline Estudo & Estudo, amostra & Variáveis avaliadas, idade & Resultados \\
\hline Lages et a/(33) & Transversal, $\mathrm{n}=78$ & ceo-d/CPO-D, 1 a 32 anos & $\begin{array}{l}\text { ceo-d/CPO-D elevados, } 86,6 \% \\
\text { gengivite e } 8 \% \text { periodontite }\end{array}$ \\
\hline Alves et al(13) & Transversal, $n=46$ & CPO-D, fissura - 15 a 33 anos & $\begin{array}{l}\text { Cárie: fissura labiopalatal, sem } \\
\text { associação com CPO-D }\end{array}$ \\
\hline Kirchberg et a/(6) & $\begin{array}{l}\text { Controlado } \\
623 \text { casos } \\
47.646 \text { controles }\end{array}$ & $\begin{array}{l}\text { ceo-d/CPO-D em crianças } \\
\text { com fissura de lábio-alvéolo- } \\
\text { palato de } 6 \text { a } 16 \text { anos }\end{array}$ & $\begin{array}{l}\text { Cárie em fissurados labial, alveolar } \\
\text { ou palatal em dentes decíduos } \\
\text { e permanentes }\end{array}$ \\
\hline Besseling e Dubois ${ }^{(8)}$ & Transversal, $\mathrm{n}=154$ & $\begin{array}{l}\text { ceo/CPO-D em crianças } \\
\text { fissuradas de } 4-6,11-13 \text { e } \\
14-16 \text { anos }\end{array}$ & $\begin{array}{l}\text { Fissurados labiopalatais uni/ou/bilateral } \\
\text { >cárie e >CPO-D que fissurados labiais/ } \\
\text { ou/labiais e alveolares }\end{array}$ \\
\hline AI-Wahadni et al/(34) & Transversal, $n=32$ & $\begin{array}{l}\text { CPO-D, doença peridontal - } \\
10-28 \text { anos }\end{array}$ & $\begin{array}{l}\text { Fissurados labiopalatais >CPO-D e } \\
\text { doença periodontal }\end{array}$ \\
\hline Montagnoli et a/ ${ }^{(2)}$ & Transversal, $\mathrm{n}=881$ & $\begin{array}{l}\text { Crescimento em fissurados - } \\
1-24 \text { meses }\end{array}$ & $\begin{array}{l}\text { Peso/altura: fissura-labial+palatal e } \\
\text { fissura palatal }\end{array}$ \\
\hline Silva et al(1) & Transversal, $n=50$ & $\begin{array}{l}\text { Aleitamento-mães-fissurados - } \\
0-5 \text { anos }\end{array}$ & $\begin{array}{l}\text { Aleitamento natural: tipo de fissura e } \\
\text { orientačoses recebidas }\end{array}$ \\
\hline Heringer et $\left.a\right|^{(27)}$ & Transversal, $n=200$ & $\begin{array}{l}\text { Amamentação, hábitos orais - } \\
5-7 \text { anos }\end{array}$ & $\begin{array}{l}\text { Tempo de amamentação natural não } \\
\text { afeta hábitos de sucção }\end{array}$ \\
\hline Souza et al(20) & Transversal, $\mathrm{n}=79$ & $\begin{array}{l}\text { Alimentação, sucção, } \\
\text { oclusão }-2-5 \text { anos }\end{array}$ & $\begin{array}{l}\text { Crianças com hábitos deletérios } \\
\text { > más-oclusões }\end{array}$ \\
\hline Stec et al(31) & Transversal, $\mathrm{n}=100$ & $\begin{array}{l}\text { Higiene bucal, periodonto em } \\
\text { fissurados }\end{array}$ & $\begin{array}{l}\text { Má higiene bucal: } 57 \% \text { e >escores } \\
\text { biofilme: na fissura }\end{array}$ \\
\hline Gimenez et $a^{(15)}$ & Transversal, $n=226$ & $\begin{array}{l}\text { Má oclusão, aleitamento - } \\
2-4 \text { anos }\end{array}$ & $\begin{array}{l}\text { >50\% má oclusão e correlação + } \\
\text { amamentação natural }\end{array}$ \\
\hline Mutarai et al(7) & Transversal, $n=138$ & $\begin{array}{l}\text { Cárie em fissurados de } \\
18-36 \text { meses }\end{array}$ & $\begin{array}{l}\text { > cárie, sem influência da fissura, } \\
\text { hábitos alimentares pobres }\end{array}$ \\
\hline Perdikogianni et a/(32) & $\begin{array}{l}\text { Controlado } \\
41 \text { casos } \\
41 \text { controles }\end{array}$ & $\begin{array}{l}\text { Condição periodontal de } \\
\text { fissurados de } 4-18 \text { anos }\end{array}$ & $\begin{array}{l}\text { Dentes na fissura >profundidade- } \\
\text { sondagem e mobilidade e >bactérias } \\
\text { periodontopatogênicas }\end{array}$ \\
\hline Al-Dajani ${ }^{(9)}$ & $\begin{array}{l}\text { Controlado } \\
53 \text { casos } \\
53 \text { controles }\end{array}$ & $\begin{array}{l}\text { Cárie dental avaliada pelo } \\
\text { índice CPO-D em fissurados } \\
\text { de } 12-29 \text { anos }\end{array}$ & $\begin{array}{l}\text { Associação: cárie e presença de fissura } \\
\text { labial e/ou palatal } \\
\text { CPO-D > nos fissurados } \\
\text { comparados ao controle }\end{array}$ \\
\hline Zhu et al(10) & $\begin{array}{l}\text { Controlado } \\
380 \text { casos } \\
339 \text { controles }\end{array}$ & $\begin{array}{l}\text { ceo-d/CPO-D: fissuradas de } \\
3-25 \text { anos }\end{array}$ & $\begin{array}{l}\text { ceo-d/CPO- } D \text { fissurados-palatais } \mathrm{e} \\
\text { fissurados labiais e/ou palatais têm mais } \\
\text { cárie que crianças da mesma idade }\end{array}$ \\
\hline
\end{tabular}

ceo-d: índice de dentes cariados, extraídos e obturados; CPO-D= índice de dentes cariados, perdidos e obturados. 
A alimentação de 60 crianças portadoras de lesão labiopalatal, de dois a quatro anos de idade e de ambos os sexos, foi estudada quanto ao tipo de aleitamento e à introdução alimentar do nascimento ao primeiro ano de vida. Os autores verificaram que, apesar da malformação, as crianças conseguiram aleitar ao seio nos primeiros meses de vida ${ }^{(14)}$.

As dificuldades de alimentação resultantes da malformação labiopalatal ou da incapacidade de absorção de nutrientes durante os primeiros meses de vida são fatores que causam déficit de crescimento em crianças com essas malformações. Pesquisadores $^{(2)}$ analisaram o crescimento de 881 crianças, de ambos os sexos até o segundo ano de vida com três tipos de fissuras. Concluíram que o comprometimento do peso e do comprimento foi mais grave nos lactentes com fissura labiopalatal e fissura palatal. Esse fato foi atribuído ao defeito conjunto de lábio e/ou palato, o qual amplia as dificuldades de alimentação, em comparação ao grupo com fissura labial.

Os métodos de alimentação utilizados em 50 recémnascidos com fissura labiopalatal foram avaliados durante a internação e após a alta hospitalar e, em especial, a experiência das mães desses bebês em relação ao aleitamento materno. $\mathrm{O}$ aleitamento natural foi mais satisfatório em crianças com fissura menos complexa, do tipo pré-forame incisivo, e a sucção insuficiente foi a principal causa de substituição do aleitamento natural por outras formas de alimentação ${ }^{(1)}$.

Assim sendo, os métodos de alimentação necessários para recém-nascidos com fissura de lábio e/ou palato são basicamente os mesmos adotados para outros recém-nascidos sem fissura de lábio e/ou palato, observando o tempo de amamentação e a posição ao seio ${ }^{(23)}$. As crianças portadoras de fissura pré-forame incisivo não têm problemas alimentares, mas aquelas com fissuras pós-forame ou transforame incisivo podem apresentar dificuldades alimentares por não conseguirem uma pressão intrabucal adequada ${ }^{(3)}$.

$\mathrm{O}$ aleitamento materno oferece ao bebê um adequado desenvolvimento ósseo e muscular, garantindo, assim, a inter-relação entre o sistema estomatognático e os demais órgãos e funções.

\section{Adequado manuseio no processo de aleitamento artificial}

Para o desenvolvimento do sistema estomatognático, a recomendação da Organização Mundial da Saúde (OMS) (24) é manter o aleitamento exclusivo até o sexto mês de vida. A falta da amamentação constitui-se num dos principais fatores etiológicos das más oclusões dentárias. Do mesmo modo, a deglutição, a fonação e a respiração podem ser afetadas quando a mamadeira é logo introduzida nos hábitos do bebê( ${ }^{(25)}$.

$\mathrm{O}$ aleitamento artificial com o leite materno, de vaca ou com leites industrializados, deve ser feito com colher. A mamadeira é oferecida somente quando se esgotarem todas as tentativas para se estabelecer o aleitamento natural. Nesse caso, pode-se usar a mamadeira com bico ortodôntico, com um ou três furos pequenos na região anterior, voltado para cima $^{(23)}$. A quantidade de leite a ser mamada deve acompanhar o grau de saciedade e desenvolvimento pônderoestatural da criança. A amamentação artificial satisfaz às necessidades nutricionais da criança, porém grande parte das excitações, que partem da boca, presentes na amamentação natural, fica anulada ${ }^{(26)}$. O fluxo da mamadeira não incentiva os movimentos de protrusão e retrusão; a língua fica parada, realizando apenas um movimento de "vai e vem", funcionando como uma "válvula" que irá impedir que o bebê se afogue. Mesmo com um orifício pequeno, o trabalho muscular será deficiente, sendo os músculos mastigatórios pouco exigidos. Os músculos bucinadores irão se desenvolver de forma predominante ${ }^{(16)}$. O crescimento e o desenvolvimento normal da face podem ser prejudicados pelo formato dos bicos de mamadeira ${ }^{(27)}$. Na escolha de um bico adequado, deve-se considerar o comprimento e a flexibilidade, bem como o tamanho do furo e a posição que o mesmo irá impor na cavidade bucal. O comprimento do bico da mamadeira não pode interferir no crescimento da face, como os bicos curtos. O bico deve ser flexível o suficiente para permitir uma fácil adaptação à boca da criança, assim como o furo precisa favorecer um adequado fluxo de leite.

Em situações nas quais se necessita de uma alternativa à mamadeira, a alimentação com xícara proporciona uma solução simples e efetiva. O bebê pode regular sua própria ingestão alimentar, de maneira que o leite somente toque seus lábios e não seja despejado dentro da boca. A respiração torna-se mais fácil de ser controlada e a deglutição ocorre quando o bebê está apto para essa função. Como benefício, evita-se a aspiração, tão comum entre as crianças portadoras de fissuras de lábio e/ou palato ${ }^{(3)}$. Tanto o aleitamento materno como o uso de xícaras ou mamadeira podem não ser possíveis quando a má formação palato-labial é extensa, sendo necessário usar uma seringa para alimentar o bebê. Assim, colocando-se um tubo fino ao lado do mamilo, o qual irá liberar o leite materno previamente ordenhado, o bebê pode ter a sensação de estar mamando no peito ${ }^{(3)}$.

$\mathrm{O}$ uso de placas palatais obturadoras funcionam como palato artificial, servindo de apoio para a criança pressionar 
o bico com a língua durante a sucção ${ }^{(1,22)}$. Em contraposição, há contraindicações ${ }^{(1,28)}$, pois elas poderiam deformar e direcionar o crescimento da maxila se não forem modeladas e substituídas com o desenvolvimento da criança.

\section{Relação entre amamentação e estado de saúde bucal}

O estado de saúde bucal é influenciado pela higiene oral da criança fissurada. Os pacientes portadores de fissura labiopalatal precisam de tratamento dentário preventivo desde o primeiro ano de vida. O exame clínico do bebê deve ocorrer antes mesmo da erupção dos primeiros dentes decíduos. A higiene bucal precisa ser realizada diariamente para remover os restos alimentares e também para que a criança se habitue ao manuseio da cavidade bucal, especialmente na região da falha. A gengiva, bochecha, língua e palato do bebê devem ser limpos com dedeira, gaze ou fralda embebida em água filtrada ou em soro fisiológico. O mesmo procedimento deve ser adotado após a erupção do primeiro dente ${ }^{(29)}$.

As crianças com fissuras orais têm um maior risco de desenvolvimento de cárie na dentição decídua do que as crianças da mesma idade sem a malformação ${ }^{(6,8-10)}$. Aspectos dentais são primordiais para uma reabilitação bem sucedida da fenda ${ }^{(25)}$. Os fatores que influenciam o início da doença cárie foram avaliados em 69 crianças portadoras de fissura de lábio e/ou

\section{Referências bibliográficas}

1. Silva $E B$, Fúria $C L$, Di Ninno $C Q$. Aleitamento materno em recém-nascidos portadores de fissura labiopalatina: dificuldades e métodos utilizados. Rev CEFAC 2005;7:21-8.

2. Montagnoli LC, Barbieri MA, Bettiol H, Marques IL, Souza L. Prejuízo no crescimento de crianças com diferentes tipos de fissura lábio-palatina nos 2 primeiros anos de idade. Um estudo transversal. J Pediatr (Rio J) 2005;81:461-5

3. Araruna RC, Vendrúsculo DM. Alimentação da criança com fissura de lábio e/ou palato - um estudo bibliográfico. Rev Latino-Am Enfermagem 2000;8:99-105.

4. World Health Organization [homepage on the Internet]. Breastfeeding key to saving children's lives - WHO Technical Report Series, 2010 [cited 2011 Feb 10]. Available from: http://www.who.int/mediacentre/news/notes/2010/ breastfeeding_20100730/en/

5. Santos GG. Padrões de fala de indivíduos com fissura lábio-palatina: análise pré e pós-cirúrgica [tese de mestrado]. Curitiba (PR): Universidade Tuiuti do Paraná; 2000.

6. Kirchberg A, Treide A, Hemprich A. Investigation of caries prevalence in children with cleft lip, alveolus, and palate. J Craniomaxillofac Surg 2004;32:216-9.

7. Mutarai T, Ritthagol W, Hunsrisakhun J. Factors influencing early childhood caries of cleft lip and/ou palate children aged 18 to 36 months in southern Thailand. Cleft Palate Craniofac J 2008;45:468-72.

8. Besseling S, Dubois L. The prevalence of caries in children with a cleft lip and/ or palate in Southern Vietnam. Cleft Palate Craniofac J 2004;41:629-32.

9. Al-Dajani M. Comparison of dental caries prevalence in patients with cleft lip and/ or palate and their sibling controls. Cleft Palate Craniofac J 2009;46:529-31. palato, com idade entre 18 e 36 meses. As crianças portadoras de fissura de lábio e/ou palato apresentaram um número maior de cáries, além de hábitos alimentares menos saudáveis. Os responsáveis por essas crianças tinham menor conhecimento e comportamento adequado em relação à saúde bucal. A presença da fissura não foi fator importante para a prevalência de cárie e, sim, o hábito alimentar com amamentação artificial noturna e consumo frequente de alimentos açucarados, sendo estes associados significativamente à cárie dentária ${ }^{(7)}$.

Além dos problemas bucais relacionados à cárie dental, a criança nascida com fissura de lábio e/ou palato, devido a anormalidades dentofaciais e ortodônticas, pode apresentar doença periodontal ${ }^{(29-34)}$, podendo interferir na alimentação da criança.

\section{Conclusão}

O aleitamento natural em crianças portadoras de fissuras de lábio e/ou palato, é fator decisivo para a correta maturação e crescimento craniofacial em nível ósseo, muscular e funcional, como também na prevenção de problemas bucais. Além disso, essas crianças apresentam maior prevalência de cárie dental, e doença periodontal, podendo ainda apresentar problemas de má oclusão.

10. Zhu WC, Xiao J, Liu Y, Wu J, Li JY. Caries experience in individuals with cleft lip and/or palate in China. Cleft Palate Craniofac J 2010;47:43-7.

11. Vasconcelos NP, Melo P, Gavinha S. Estudo dos factores etiológicos das cáries precoces da infância numa população de risco. Rev Port Estomatol Cir Maxilofac 2004;45:69-77.

12. Bratthall $D$, Hansel Petersson G. Cariogram--a multifactorial risk assessment model for a multifactorial disease. Community Dent Oral Epidemiol 2005;33:256-64

13. Alves LM, Melo GG, Pereira JR, Cardoso MS. Prevalência de cárie em portadores de fissura lábio-palatais atendidos no Instituto Materno Infantil de Pernambuco. Odontol Clin-Científ 2004;3:57-60.

14. Pini JG, Peres SP. Alimentação do lactente portador de lesão lábio-palatal: aleitamento e introdução alimentar. Rev Nutr 2001;14:195-9.

15. Gimenez CM, Moraes AB, BertozAP, Bertoz FA, Ambrosano GB. Prevalência de más oclusões na primeira infância e sua relação com as formas de aleitamento e hábitos infantis. R Dental Press Ortodon Ortop Facial 2008;13:70-83.

16. Bervian J, Fontana M, Caus B. Relação entre amamentação, desenvolvimento motor bucal e hábitos bucais - revisão de literatura. RFO 2008;13:76-81.

17. Azeredo CM, Maia TM, Rosa TC, Silva FF, Cecon PR, Cotta RM. Percepção de mães e profissionais de saúde sobre o aleitamento materno: encontros e desencontros. Rev Paul Pediatr 2008;26:336-44.

18. Nassar E, Marques IL, Trindade-Júnior AS, Bettiol H. Feeding-facilitating techniques for the nursing infant with Robin sequence. Cleft Palate Craniofacl J 2006;43:55-60.

19. Antunes LS, Antunes LA, Corvino MP, Maia LC. Amamentação natural como fonte de prevenção em saúde. Cienc Saude Coletiva 2008;13:103-9. 
20. Souza DF, Valle MA, Pacheco MC. Relação clínica entre hábitos de sucção, má oclusão, aleitamento e grau de informação prévia das mães. R Dental Press Ortodon Ortop Facial 2006;11:81-90.

21. McLeod NM, Urioste ML, Saeed NR. Birth prevalence of cleft lip and palate in Sucre, Bolivia. Cleft Palate Craniofac J 2004;41:195-8.

22. Tolarova MM. Cleft lip and palate: treatment. e-medicine World Medical Library [serial on the Internet]. 2009 [cited 2010 Mar 29]. Available from: http:// emedicine.medscape.com/article/995535-treatment

23. Andrade IS, Guedes ZC. Sucção do recém-nascido prematuro: comparação do método Mãe-Canguru com os cuidados tradicionais. Rev Bras Saude Matern Infant 2005;5:61-9.

24. Organização Mundial da Saúde (OMS). Recomendações da Organização Mundial da Saúde a respeito de aleitamento materno [serial on the internet]. 2010 [cited 2010 Mar 18]. Available from: http://www.unicef.org/programme/ breastfeeding/baby.htm

25. Marques RF, Lopez FA, Braga JA. O crescimento de crianças alimentadas com leite materno exclusivo nos primeiros 6 meses de vida. J Pediatr (Rio J) 2004;80:99-105.

26. Medeiros EB, Rodrigues MJ. A importância da amamentação natural para o desenvolvimento do sistema estomatognático do bebê. Rev Cons Reg Odontol Pernamb 2001;4:79-83.

27. Heringer MR, Reis M, Pereira LF, Di Ninno CQ. A influência da amamentação natural no desenvolvimento dos hábitos orais. Rev CEFAC 2005;7:307-10.
28. Turner L, Jacobsen C, Humenczuk M, Singhal VK, Moore D, Bell H. The effects of lactation education and a prosthetic obturator appliance on feeding efficiency in infants with cleft lip and palate. Cleft Palate Craniofac J 2001;38:519-24.

29. Ribeiro-Roda S, Gil-da-Silva-Lopes VL. Aspectos odontológicos das fendas labiopalatinas e orientações para cuidados básicos. Rev Cienc Med 2008; 17:95-103.

30. American Cleft Palate-Craniofacial Association [homepage on the Internet] Parameters for evaluation and treatment of patients with cleft lip/palate or other craniofacial anomalies. Chapel Hill NC: The Maternal and Child Health Bureau, Title V, Social Security Act, Health Resources and Services Administration, US Public Health Service, DHHS [serial on the internet]. 2007 [cited $2010 \mathrm{Apr}$ 1]. Available from: http://www.acpa-cpf.org/teamcare/parameters $07 \mathrm{rev} . p d f$

31. Stec M, Szczepańska J, Pypeć J, Hirschfelder U. Periodontal status and oral hygiene in two populations of cleft patients. Cleft Palate Craniofac J 2007;44:73-8

32. Perdikogianni H, Papaioannou W, Nakou M, Oulis C, Papagiannoulis L. Periodontal and microbiological parameters in children and adolescents with cleft lip and /or palate. Int J Paediatr Dent 2009;19:455-67.

33. Lages EM, Marcos B, Pordeus IA. Oral health of individuals with cleft lip, cleft palate, or both. Cleft Palate Craniofac J 2004;41:59-63.

34. Al-Wahadni A, Alhaija EA, Al-Omari MA. Oral disease status of a sample of Jordanian people ages 10 to 28 with cleft lip and palate. Cleft Palate Craniofac J 2005;42:304-8. 